\title{
Vernetzte Depressionsbehandlung Der Hausarzt kann die Weichen stellen
}

Hans-Günther Hofmann

Hausärztlich niedergelassener Facharzt für Innere Medizin, Mainz-Bretzenheim

Depressionen sind eine der häufigsten und schwersten Erkrankungen. Sie sind nicht nur mit einer hohen Rückfallquote, sondern auch einem hohen Sterberisiko verbunden. Jeder zehnte Patient im Wartezimmer einer hausärztlichen Praxis leidet unter einer Depression. Leider wird die Diagnose oft nicht gestellt und somit den Patienten eine zielgerichtete Therapie vorenthalten. Nach einer Studie der Weltgesundheitsorganisation WHO (5) gilt dies nicht nur weltweit, sondern auch in der Bundesrepublik Deutschland (8). In der Studie wurde nachgewiesen, dass die unipolaren Depressionen die größte Bedeutung unter den Volkskrankheiten der entwickelten Länder haben ${ }^{1}$, die bipolare Störung rangierte danach immerhin auf Platz 6!

\section{Studie "Depression 2000"}

In der Studie „Depression 2000“, in der 412 Hausarztpraxen erfasst wurden, konnte gezeigt werden, dass 10,9\% aller Patienten unter Depressionen leiden (11,9\% der Frauen und 9,4\% der Männer) (8). Nahezu $50 \%$ dieser Depressionen wurden als schwergradig eingestuft. 51,3\% der Erkrankten hatten bereits früher mindestens eine depressive Episode. Hausärzte wurden häufiger konsultiert als Fachärzte. Bei lediglich ca. zwei Drittel wurde die Krankheit als „psychische Störung“ eingeordnet, davon immerhin 38,5\% als gesicherte Depression. Das heißt im Umkehrschluss, dass etwa ein Drittel der Depressionen als somatische Krankheit eingeordnet wurde, bei etwa zwei Drittel bestand keine Sicherheit in der Diagnose. Fast zwei Drittel der Erkrankungen wurden vom Hausarzt behandelt mit ca. 10\% unter fachärztlicher oder psychotherapeutischer Mitbehandlung. Jeder fünfte Patient wurde - meist wegen schwerer Depression sofort überwiesen, ohne dass der Hausarzt selbst behandelte.
Etwa jeder zehnte Patient einer hausärztlichen Praxis leidet unter einer Depression. Circa zwei Drittel der Erkrankten werden beim Hausarzt behandelt. Die Diagnostik ist schwer, da oft nur körperliche Beschwerden angegeben werden. Die Behandlung von Depressionen lohnt sich und mindert das Risiko von Koerkrankungen. Zur Vermeidung von Chronifizierung (lange Arbeitsunfähigkeitszeiten, Verlust des Arbeitsplatzes und Frühberentung) müssen noch neue Schnittstellen und bessere Strukturen entwickelt werden.

\section{Woraus bestand in der Studie die hausärztliche Behandlung?}

Bei der Befragung nach der Art der Therapie (Abb. 1) wurden von den an der Studie beteiligten Hausärzten eine Kombinationsbehandlung als indiziert angesehen. 70,1\% brauchen Beratung, Gespräch und Krisenintervention, 20,8\% Psychotherapie und 81,0\% Pharmakotherapie davon 58,2\% Antidepressiva, 36,4\% Phytotherapeutika, 23,8\% Sedativa/Hypnotika (in der Regel als Komedikation zu SSRI) $(1,8)$.

Auf der Kölner Tagung der AnyCare GmbH im Oktober 2007 wurde folgendes Fazit gezogen: Nur etwa die Hälfte der Depressiven sucht ärztliche Hilfe, davon ist die Hälfte nicht angemessen versorgt. Ein früher Therapieabbruch ist häufig, da sich die Beschwerden nicht binnen kurzer Zeit bessern (7).

Nach dem bundesdeutschen Survey wurden $61 \%$ der depressiven Patienten weder psychotherapeutisch noch medikamentös behandelt (8).

\section{Schlüsselrolle für den Hausarzt?}

Da viele depressive Patienten zuächst ihren Hausarzt aufsuchen, kommt diesem eine Schlüsselrolle zu (3). Etwa $20 \%$ der Patienten haben eine psychiatrische Diagnose nach ICD10 , wobei depressive Syndrome überwiegen. Auch wenn sie oft leichter ausgeprägt sind als in der fachärztlichen Behandlung, bedeuten sie für die Betroffenen eine hohe psychosoziale Beeinträchtigung (Auftreten von Komorbidität, schlechtere Prognose begleitender somatischer
${ }^{1}$ gemessen in YLD, years of live lived with disability, Lebensjahre mit Funktionsbeeinträchtigung 


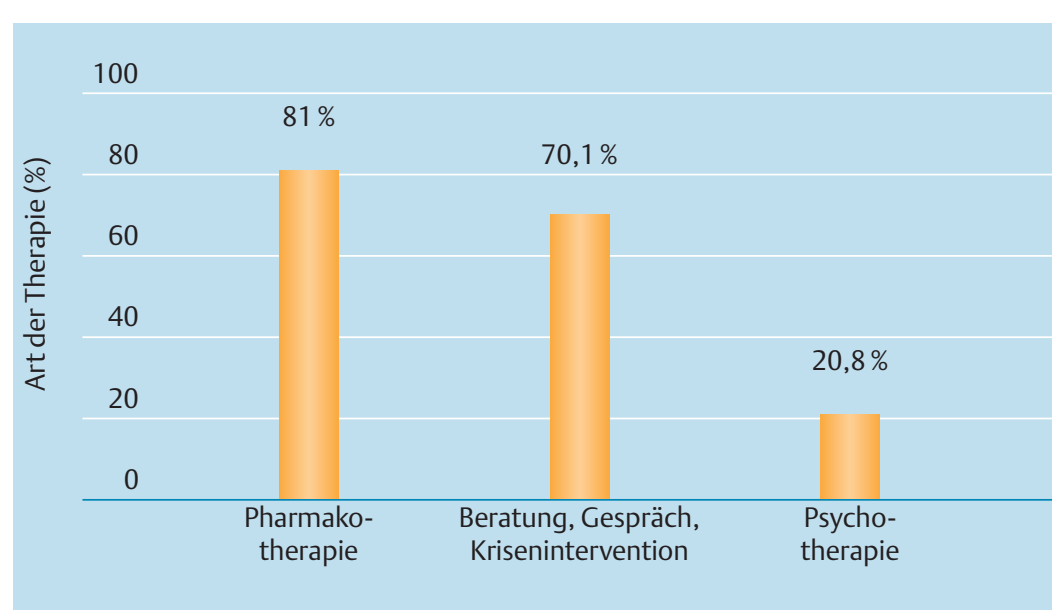

Abb. 1 Ergebnisse der Studie „Depression 2000“: Art der hausärztlichen Behandlung bei Depression.
Krankheit und trägt zur Entstigmatisierung bei. Dennoch ist immer noch eine Hürde zu überwinden, wenn man Patienten antidepressiv behandeln oder sie zum Nervenarzt bzw. zur Psychotherapie überweisen will.

\section{Die Schwierigkeit der Diagnose}

Die Diagnose ‘Depression' wird zu häufig nicht richtig gestellt. Die Patienten geben oft nur körperliche Beschwerden an („Mein Rücken schmerzt.“, „Mir ist schwindelig.“, „Ich habe Kopfschmerzen.") oder ihre Angaben sind unspezifisch („Ich bin fertig, ausgebrannt.“, „Ich habe keine Energie mehr.“). Dahinter die Depression zu erkennen, ist nicht immer leicht, genauso wenig, wie zwischen normalen psychischen Reaktionen (z.B. Trauer) und einer behandlungsbedürftigen Depression zu unterscheiden. Hilfreich könnten standardisierte Tests sein, wobei die zwei Fragen „Fühlten Sie sich im letzten Monat häufig niedergeschlagen, traurig, bedrückt oder hoffnungslos?“ und „Hatten Sie im letzten Monat deutlich weniger Lust und Freude an Dingen, die Sie sonst gerne tun?" kaum schlechter abschneiden als der WHO-5-Fragebogen zum Wohlbefinden (1). Aufwändigere Tests sind in den Ablauf einer hausärztlichen Praxis kaum einzugliedern. Eine große Hilfe ist die Broschüre „Depression - Therapieempfehlungen der Arzneimittelkommission der deutschen Ärzteschaft" (Abb. 2), in der die diagnostischen Schritte ebenso erläutert sind wie klare Regeln für die Überweisung zu einem Facharzt (1).

Genauso wie eine Depression kann auch eine körperliche Erkrankung als ihre Ursache für psychische Beschwerden übersehen werden. (Nicht umsonst werden in den Leitlinien „Affektive Erkrankungen“ der Deutschen Gesellschaft für Psychiatrie, Psychotherapie und Nervenheilkunde (DGPPN) (8) unter Punkt „4. Zusatzuntersuchungen" wichtige Hinweise auf mögliche organische Störungen gegeben). Hierbei sind vor allem Schilddrüsenfunktionsstörungen, Viruserkrankungen, Medikamente (Betablocker, Antibiotika, Kortison u.a.), Leber- und Nierenerkrankungen zu nennen.

Eine weitere Schwierigkeit besteht darin, dass die Patienten oft an meh-

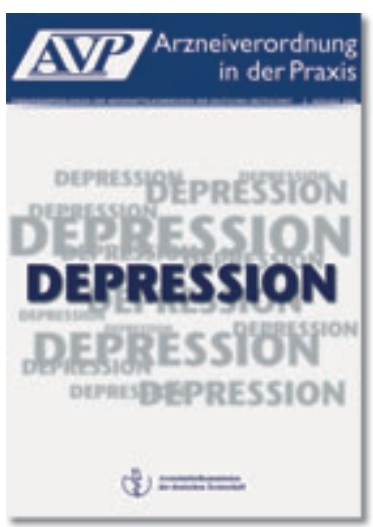

Abb. 2 Broschüre „Depression - Therapieempfehlungen der Arzneimittelkommission der deutschen Ärzteschaft. 
reren Krankheiten leiden: Der Diabetiker kann sehr wohl depressiv sein und der Depressive eine Koronare Herzkrankheit (KHK) haben. Wir müssen eine Depression ebenso aktiv suchen wie wir auch nach somatischen Krankheiten systematisch forschen. Und wir dürfen den komorbid Depressiven eine antidepressive Therapie aus Angst vor unerwünschten Arzneimittelnebenwirkungen nicht vorenthalten. Als Beispiel sei die antidepressive Therapie bei KHK-Patienten genannt. Hier wurden gerade zwei Studien veröffentlicht: Bis zu $27 \%$ der Patienten, die mit einer KHK stationär behandelt wurden, leiden an einer Depression, die zu einer 3-fach erhöhten kardialen Letalität führt. Die selektiven Serotonin-Wiederaufnahmehemmer Sertralin (SADHART-Studie) und Citalopram (CREATE-Studie) hatten keine unerwünschten kardiovaskulären Effekte und konnten wirksam bei KHK-Patienten eingesetzt werden. Es profitierten vor allem die Patienten, die schon einmal wegen einer depressiven Episode behandelt wurden $(2,4)$.

\section{Wo ist der Weg aus der Chronifizierung?}

Die Krankheit Depression ist nicht nur schwerwiegend, sie kostet die Gesellschaft auch viele Ressourcen und Geld. Psychische Erkrankungen sind die häufigste Ursache für Berufsunfähigkeit. Der TK-Gesundheitsreport aus 2005 verzeichnete nach Angaben des Verbandes Deutscher Rentenversicherter bei Männern einen Anstieg der Berufsunfähigkeit von $8 \%$ in 1983 auf fast $27 \%$ in 2004 . Bei Frauen erhöhte sich der Anteil von unter $10 \%$ auf rund $37 \%$ (1) in demselben Zeitraum. Krankschreibungen aufgrund psychischer Störungen haben bei sinkenden Gesamtzahlen in den letzten 5 Jahren um 20 \% zugenommen (TK-Gesundheitsreport 2005) (10). Der Anteil der aufgrund einer Depression arbeitsunfähigen Erwerbstätigen wuchs von 2000 bis 2004 um beachtliche $42 \%$. In 2004 waren absolut gesehen bereits $1 \%$ der Erwerbstätigen wegen Depression arbeitsunfähig (DAKGesundheitsreport 2005) (10).

Eine wichtige Aufgabe ist, eine Chronifizierung der Depression zu verhindern. Nur: Wo sind die Schnittstellen, an denen die Profis die Patienten zu ihrer Arbeit zurück begleiten - vielleicht mit weniger Arbeitszeit dafür mit mehr Verständnis für die speziellen Probleme? Wer zeigt den Patienten ihre Stärken? Wir brauchen über die bisherigen Netzwerke hinaus Kontakte u.a. über die verschiedenen Kostenträger, den Medizinischen Diensten der Krankenversicherung (MDK) und den Betriebsärzten. Es bleibt eine wichtige, eine spannende Diskussion.
Interfacial treatment of depressive disorders - The general physician can be the switchman

About one in ten patients of a practising physician suffers from depression. Approximately two thirds of these depressed patients are medicated by general practitioners. Diagnosis is difficult, since many patients merely describe physical discomfort. Treating depression is worth the effort and reduces the risk of concomitant diseases. To avoid a chronic condition (prolonged disability, loss of employment and early retirement) it will be necessary to develop new interfaces and structures.

Key words

Depression - rehabilitation - reinstatement - disability

Literatur

1 Arzneimittelkommission der deutschen Ärzteschaft, Hrsg. Depression - Therapieempfehlungen der Arzneimittelkomission der deutschen Ärzteschaft. 2 . Aufl. 2006

2 Glassman AH, Bigger JT jr. Antidepressants in coronary heart disease: SSRIs reduce depression, but do they save lives? JAMA 2007; 297: 411-412

3 Henkel V, Wambach V, Möller HJ, Hegerl U. Schlüsselrolle für den Hausarzt, Diagnostik und Therapie von Depressionen. MMW Fortschr Med 2001; 19 (Sonderheft 2): 495-501

4 Lespérance F, Frasure-Smith N, Koszycki D et al. Effects of citalopram and interpersonal psychotherapy on depression in patients with coronary artery disease: the Canadian Cardiac Randomized Evaluation of Antidepressant and Psychotherapy Efficacy (CREATE) trial. JAMA 2007; 297: 367-379

5 Murray C], Lopez AD. Global and regional descriptive epidemiology of disability: Incidence, prevalence, health expectancies and years lived with disability. In: Murray C], Lopez AD, eds. The global burden of disease. Global burden of disease and injury series. Harvard: Harvard university Press; 1997: 201-246

6 Rätsel Depression, eine Sendung von Quarks \& Co des WDR-Fernsehens vom 16. Oktober 2007. Als Podcast herunterzuladen unter www.wdr.de/tv/ quarks/sendungsbeitraege/2007/1016/001_ depression.jsp

7 Rückle S. Depressive benötigen strukturierte Betreuung - auch außerhalb von Sprechstunden. Bericht der Fachtagung der AnyDay ProPerspektive. Dtsch Med Wochenschr 2007; 132: 2473

8 Wittchen HU, Müller N, Schmidtkunz B et al. Erscheinungsformen, Häufigkeiten und Versorgung von Depressionen. Ergebnisse des bundesweiten Gesundheitssurveys „Psychische Störungen“. MMW Fortschr Med 2000; 118 (Suppl. I): 4-10

9 Wittchen HU, Müller N, Schmidtkunz B et al. Erscheinungsformen, Häufigkeiten und Versorgung von Depressionen. Ergebnisse des bundesweiten Gesundheitssurveys „Psychische Störungen“. MMW Fortschr Med 2000; 118 (Suppl. I): 22-30

10 www.buendnis-depression.de/depression/arbeitsplatz.php

11 www.uni-duesseldorf.de/awmf/II-na/038-012.htm

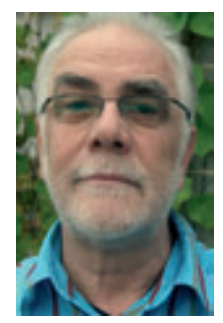

Korrespondenz

Hans-Günther Hofmann

Bert-Brecht-Straße 5

55128 Mainz-Bretzenheim

Doerflinger.Hofmann@telemed.de 